\title{
On the measure of electron correlation and entanglement in quantum chemistry based on the cumulant of the second-order reduced density matrix
}

\author{
D. R. Alcoba, ${ }^{1}$ R. C. Bochicchio, ${ }^{1, a)}$ L. Lain, ${ }^{2}$ and A. Torre ${ }^{2}$ \\ ${ }^{1}$ Departamento de Física, Facultad de Ciencias Exactas y Naturales, Universidad de Buenos Aires, Ciudad \\ Universitaria, Buenos Aires 1428, Argentina and Instituto de Física de Buenos Aires, Consejo Nacional \\ de Investigaciones Científicas y Técnicas, Ciudad Universitaria, Buenos Aires 1428, Argentina \\ ${ }^{2}$ Departamento de Química Física, Facultad de Ciencia y Tecnología, Universidad del País Vasco, \\ Apdo. 644, Bilbao E-48080, Spain
}

(Received 15 July 2010; accepted 29 September 2010; published online 11 October 2010)

In this paper we propose a functional of the many-body cumulant of the second-order reduced density matrix within the spin-free formalism of quantum chemistry which quantifies the idea of electron correlation and allows one to detect spin entanglement. Its properties are rigorously stated and discussed for spin-adapted pure states. Numerical determinations are performed for both equilibrium conformations and dissociation processes in molecular systems. (C) 2010 American Institute of Physics. [doi:10.1063/1.3503766]

\section{INTRODUCTION}

The collective behavior of an interacting many-electron quantum system is known as correlated motion and is described according to the level of approximation used for the wave function. In such systems the relevant interaction which drives the motion is that arising from the electrostatic Coulomb potential between the electrons. The differences between the description of the systems at the independent particle level of approximation (uncorrelated wave functions), that is, the Hartree-Fock (HF) solutions which corresponds to a Slater determinant and those from wave functions which explicitly incorporate the treatment of the electron interaction ${ }^{1}$ is noted in practice by the behavior of the physicochemical properties. Consequently, the correlation or many-body effects are crucial for determining the properties of atoms and molecules. ${ }^{2,3}$ Their influence on the electron distribution reveals through their relationships with the chemical descriptors and the topology of the distribution $^{4,5}$ and it is crucial in the understanding of the nature of the chemical bond. ${ }^{6-19}$ The fundamental chemical concepts are the summary of the physical information contained in the pth-order reduced density matrices (p-RDMs) of an $N$-electron molecular system $(\mathrm{p}<N)$ which are directly derived from the wave function. ${ }^{2,3,10}$ Most of the attempts to describe the electron distribution in molecular systems have been concentrated on the spin blocks of the first-order reduced density matrices (spin up or $\alpha$ and spin down or $\beta$ 1-RDMs), which determine electron and spin densities and provide intuitive interpretations of chemical data. ${ }^{3,10,11} \mathrm{Nev}$ ertheless, electron densities are not enough for a complete description of the electron distribution of molecular systems, especially those undergoing chemical reactions and electron rearrangements (nonequilibrium geometrical conformations) such as formation and breaking of bonds. In these cases,

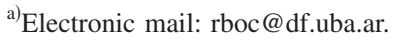

higher-order density matrices are relevant ${ }^{20-23}$ and particularly the second-order reduced density matrices (2-RDM) are essentially needed. ${ }^{3,10}$ These latter types of system states permit to introduce a natural linkage between the quantum chemistry phenomena of correlation and entanglement ${ }^{23}$ (the correlation that has not a classical counterpart). This linkage is based on electron interaction and spin conservation in a single molecular system during the rearrangement.

In spite of the central place the electron correlation plays in the electron distribution description, this is not an observable in the quantum mechanical sense and consequently, there is not a unique way to quantify its strength. Therefore the search for a measure or degree of correlation (correlation strength) associated with a determined wave function is a challenge in quantum chemistry. The most widespread way to perform this task rises from its definition and refers to the difference between the actual and the HF energy., ${ }^{2,3}$ There are also measures of electron correlation, such as the statistical correlation coefficients based on the structure of the reduced density matrices ${ }^{24,25}$ and recently others supported by entropic concepts. ${ }^{20,21,23}$ Entanglement definition shares the same difficulty and may be defined according to physical considerations. Therefore, to give a measure of entanglement is related with the idea of quantifying the correlation (its nonclassical component) of the molecular electronic states, which simplest example in quantum mechanics is the singlet state of two spins. ${ }^{26}$ Antecedents in this sense may be found in Ref. 22, where a Frobenius norm of the spin-dependent cumulant matrix has been proposed to provide a quantitative measure of correlation from spin entanglement of the state; up to what we know it is the first definition in the scenario of the two-particle reduced density matrix. Another interesting approach based on entropic concepts to define entanglement in molecular systems has been proposed in Ref. 23. We will adopt the definition based on the general statement that a quantum system of $N$ identical particles in a pure state con- 
tains at least two nonentangled subgroups of particles when all the subgroups exhibit a complete set of properties. ${ }^{27,28}$ This definition intuitively corresponds to the physical situation of processes such as the mentioned electron rearrangements during chemical reactions; dissociation processes are particular cases of central importance in understanding the nature of chemical bond. ${ }^{29}$ In such cases, it is necessary to specify the pure states of the system as being spin-adapted, i.e., the wave functions are eigenfunctions of the squaredand projection-spin operators, $\hat{S}^{2}$ and $\hat{S}_{z}$, with eigenvalues $S(S+1)$ and $S_{z}$, respectively. We will restrict ourselves to such kind of states because spin purity is of fundamental importance for a correct interpretation of highly correlated entangled states, despite in general a pure state may not be necessarily spin-adapted ${ }^{20,21}$ for most of cases in which entanglement is defined. Regarding these previous considerations, we propose a new measure of electron correlation in molecular electronic states based on the many-body spin-free cumulant of the 2-RDM which collects spin and Coulomb correlation effects and allows one to quantity spin entanglement. The onset of this measure has its physical roots in the concept of effectively unpaired electrons (particle-hole interrelations $)^{18,30,31}$ which gathers correlation effects in both closed- and open-shell systems. It is shown that the definition proposed follows the necessary physical and mathematical conditions expected to describe phenomena related with molecular electron distribution.

We have organized this article as follows. The second section summarizes the theoretical concepts and notation used in this paper. In the third section we propose the new tool for measuring electron correlation and spin entanglement, showing its advantages. Our proposal has been applied to selected molecular systems in equilibrium and nonequilibrium conformations; a discussion of the obtained numerical results and their physical consequences is reported in the fourth section. Finally, the last section points out the conclusions of this work.

\section{THEORETICAL BACKGROUND}

We will describe an $N$-electron system by means of a determined wave function, $\Psi\left(S, S_{z}\right)$, with defined spin quantum numbers $S$ and $S_{z}$. The elements of the first- and secondorder reduced density matrices corresponding to that state will be denoted by ${ }^{1} D_{j^{\sigma}}^{i^{\sigma}}$ and ${ }^{2} D_{j^{i^{\sigma}} l^{\sigma^{\prime}}}^{i^{\sigma} \sigma^{\sigma^{\prime}}}$, respectively, in which $i, j, k, l, \cdots$ are orbitals of an orthogonal basis set and $\sigma$ and $\sigma^{\prime}$ are the spin coordinates $\left(\sigma, \sigma^{\prime}=\alpha, \beta\right)$. The spin-free version of the elements of these reduced density matrices are ${ }^{1} D_{j}^{i}=\Sigma_{\sigma}{ }^{1} D_{j^{\sigma}}^{i^{\sigma}}$ and ${ }^{2} D_{j l}^{i k}=\Sigma_{\sigma, \sigma^{\prime}}{ }^{2} D_{j^{\sigma_{l} \sigma^{\prime}}}^{i^{\sigma k^{\sigma^{\prime}}}}$ and their traces, $\operatorname{Tr}\left({ }^{1} D\right)$ and $\operatorname{Tr}\left({ }^{2} D\right)$, will be normalized to $N$ and $\left(\begin{array}{c}N \\ 2\end{array}\right)$, respectively.

The elements ${ }^{2} D_{j^{\sigma} l^{\sigma^{\prime}}}^{i^{\sigma} \sigma^{\sigma^{\prime}}}$ have been formulated by cumulant theory as ${ }^{25,32-34}$

$$
{ }^{2} D_{j}^{i{ }^{\sigma} l^{\sigma^{\prime}}}=\frac{1}{2}{ }^{\sigma^{\sigma^{\prime}}} D_{j}^{i^{\sigma}}{ }^{1} D_{l^{\sigma^{\prime}}}^{k^{\sigma^{\prime}}}-\frac{1}{2}{ }^{1} D_{l^{\sigma^{\prime}}}^{i^{\sigma}}{ }^{1} D_{j}^{k^{\sigma^{\prime}}}+\frac{1}{2} \Delta_{j}^{i{ }^{\sigma} \sigma^{\sigma^{\prime}}},
$$

where $\Delta_{j}^{i}{ }_{j}^{\sigma^{\sigma} l^{\sigma^{\prime}}}$ are the cumulant matrix elements of the secondorder reduced density matrix. The spin-free version of the elements of the second-order reduced density matrix, ${ }^{2} D_{j l}^{i k}$ $=\sum_{\sigma, \sigma^{\prime}}^{2} D_{j^{\sigma_{l}} \sigma^{\sigma^{\prime}}}^{i^{\sigma} \sigma^{\sigma^{\prime}}}$, can be written as ${ }^{35}$

$$
{ }^{2} D_{j l}^{i k}=\frac{1}{2}{ }^{1} D_{j}^{i 1} D_{l}^{k}-\frac{1}{4}{ }^{1} D_{l}^{i 1} D_{j}^{k}-\frac{1}{4}{ }^{1} D_{l}^{(s) i}{ }_{l}^{1} D_{j}^{(s) k}+\frac{1}{2} \Gamma_{j l}^{i k},
$$

in which ${ }^{1} D_{j}^{i}=\sum_{\sigma}{ }^{1} D_{j^{\sigma}}^{i^{\sigma}}$ are the elements of the spin-free firstorder reduced density matrix; ${ }^{1} D^{(s) i}={ }_{j}^{1} D_{j^{\alpha}}^{i^{\alpha}}-{ }^{1} D_{j^{\beta}}^{i^{\beta}}$ are the elements of the spin density matrix and $\Gamma_{j l}^{i k}=\sum_{\sigma, \sigma^{\prime}} \Delta_{j}^{i \sigma^{\sigma} \sigma^{\prime}}$. The elements ${ }^{2} D_{j l}^{i k}$ and ${ }^{1} D_{j}^{i}$ are independent of the spin substates of quantum number $S_{z}$ corresponding to the state $\Psi,{ }^{36}$ as well as those of the sum $\left(-(1 / 4){ }^{1} D^{(s) i}{ }_{l}^{1} D_{j}^{(s) k} ;+(1 / 2) \Gamma_{j l}^{i k}\right)$ in Eq. (2). ${ }^{35}$ However, the terms ${ }^{1} D^{(s) i}{ }_{l}^{1} D^{(s) k}$ and $\Gamma_{j l}^{i k}$ separately are $S_{z}$-dependent. ${ }^{35}$ In fact, the sum $\left(-(1 / 4){ }^{1} D^{(s) i}{ }_{l}^{1} D^{(s) k}\right.$ $\left.+(1 / 2) \Gamma_{j l}^{i k}\right)$ is the spin-free version of the cumulant of the second-order reduced density matrix..$^{25,37,38}$ The matrix $\Gamma$ possesses the fundamental property that is a size-consistent quantity. $^{22,39,40}$ This matrix may be contracted in two different ways as has been published elsewhere $e^{14,30,41}$ and it therefore shows its connection with the elements of the density matrix of effectively unpaired electrons, $u_{j}^{i}$, defined as $^{16-18,31,42}$

$$
u_{j}^{i}=\sum_{k}{ }^{1} D_{k}^{i}{ }^{1} \bar{D}_{j}^{k}=2{ }^{1} D_{j}^{i}-\sum_{k}{ }^{1} D_{k}^{i 1} D_{j}^{k},
$$

where ${ }^{1} \bar{D}_{j}^{k}$ stands for the matrix elements of the first-order hole reduced density matrix, ${ }^{1} \bar{D}$. The trace of the $u$ matrix has been termed as number of electrons effectively unpaired $^{42-44}$ so that $N_{u}=\sum_{i} u_{i}^{i}=2 N-\sum_{i, k}{ }^{1} D_{k}^{i}{ }^{1} D_{i}^{k}$.

Recently, in Refs. 30 and 45 we have reported the relationships between the quantities $N_{u}, \sum_{i, k} \Gamma_{k i}^{i k}\left(S_{z}\right)$, and the spin quantum numbers $S$ and $S_{z}$ resulting

$$
N_{u}=2 S+2 S^{2}-2 S_{z}^{2}+\sum_{i, k} \Gamma_{k i}^{i k}\left(S_{z}\right) .
$$

Note that the quantity $N_{u}$ is $S_{z}$-independent but the elements $\Gamma_{j l}^{i k}$ depend on that substate ${ }^{35}$ which is stressed by the notation $\left(S_{z}\right)$ in formula (4). In the case of the highest projection substate, $S_{z}=S$, Eq. (4) is transformed into the simpler expression

$$
N_{u}=2 S+\sum_{i, k} \Gamma_{k i}^{i k}\left(S_{z}=S\right)
$$

which provides the decomposition of the number of effectively unpaired electrons into two terms. The first term $2 S$ represents the number of net spin unpaired electrons whereas the second one $\sum_{i, k} \Gamma_{k i}^{i k}\left(S_{z}=S\right)$ accounts for the statistically irreducible correlation effects. ${ }^{30}$ The decomposition of the trace of the effectively unpaired electron density matrix $^{14,18,19,31,46,47}$ into well defined terms ${ }^{30}$ [cf. Eqs. (4) and (5)] permitted to understand the physical meaning of the cumulant densities and therefore the direct connection of the former matrix with the spin and correlation effects. Therefore because of the apparent relation between the correlation effects and the concept of entanglement, in the next section we propose an alternative assessment of electron correlation and spin entanglement based on the quantity $\Sigma_{i, k} \Gamma_{k i}^{i k}\left(S_{z}=S\right)$. 


\section{A MEASURE OF THE CORRELATION: SPIN ENTANGLEMENT CONTRIBUTION}

It has been reported that correlation measures only depending on the 1-RDM exhibit the same degree of correlation for systems states differing significantly, ${ }^{22}$ which is obviously unphysical. However, in quantum systems with twoparticle interactions, there is a one to one mapping between the 2-RDM and the wave function for nondegenerate ground states ${ }^{48}$ or for degenerate states which can be distinguished by at least one operator containing just two-particle interactions. ${ }^{33}$ Hence, Ref. 22 concluded from this mapping that the 2-RDM is needed for a correlation measure of atomic and molecular systems in order to avoid the above mentioned drawback. For these reasons and according to our previous studies involving the quantities defined by Eqs. (4) and (5), formulated in terms of many-body cumulant elements (the statistically irreducible part of the $2-\mathrm{RDM}),{ }^{30}$ it seems feasible to propose the quantity

$$
\epsilon=\sum_{i, k} \Gamma_{k i}^{i k}\left(S_{z}=S\right)
$$

as a simpler measure of electron correlation $\epsilon$, corresponding to the wave function $\Psi\left(S, S_{z}=S\right)$.

Our proposal is based on the properties of the cumulant matrix, but in practical terms it only needs the spin-free firstorder reduced density matrix (available in most of the codes) and the spin $S$ of the state of the system. However, it is worthy to note that the $\epsilon$ quantity implicitly depends on the 2-RDM due to its dependence on the $S$ spin number coming from the square spin operator $\hat{S}^{2}$, which is a two-particle operator. The quantity $\epsilon$ viewed as $N_{u}-2 S$ [Eq. (5)] should be regarded as the effective number of correlated electrons. ${ }^{21}$ It is evident from $\epsilon$ definition [cf. Eq. (6)] that this quantity accounts for the total electronic correlation which includes classical correlation and nonclassical one (i.e., entanglement). Both effects are present in the many-body cumulant part of the 2-RDM and they may not be separated. Nevertheless, as will be shown later, it is possible to evaluate the spin entanglement itself in certain cases, as in dissociation processes of molecular systems. Thus, the difference between the $\epsilon$ quantities of a given molecular system at the dissociation limit and those of its dissociation fragments allows one to extract the spin entanglement measure from the whole electronic correlation.

Let us now enumerate and discuss the properties of the spin-free 2-RDM cumulant crossed trace measure (CCTM) defined by Eq. (6). These properties constitute often required criteria for correlation and entanglement measures. ${ }^{49}$ They follow from those of the cumulant part of the 2-RDM, and hence, they are generally shared by the measure introduced in Ref. 22, the square of the Frobenius norm of the cumulant 2-RDM.

(1) CCTM is invariant under any unitary transformation of the orbital space.

(2) The correlation energy is commonly accepted to be negative; hence the negative this energy is, the more correlation the system has, defining a trend. Therefore, due to the relationship between correlation and en- tanglement concepts, measures of entanglement (and/or correlation index) have been considered as real values in the literature, i.e., the index may be positive or negative. CCTM is positive semidefinite, i.e., $\epsilon \geq 0,{ }^{30}$ the equality being valid only for a Slater determinant wave function. Also it has an upper limit, $\epsilon \leq 2(N-S) .{ }^{30}$ Consequently, such an index is easier to interpret regarding its zero reference value corresponding to the independent particle model as a nonentangled state. ${ }^{21}$

(3) CCTM for particles and holes are coincident, i.e., $\epsilon$ $=\bar{\epsilon}=\sum_{i, k} \bar{\Gamma}_{k i}^{i k}\left(S_{z}=S\right)$ as a consequence of the coincidence of the second-order particle and hole reduced density matrix cumulants, ${ }^{50}$ thus preserving the particle-hole symmetry [cf. Eq. (3)].

(4) Assuming a closed-shell system $X_{M}$ with associated spin number $S_{X_{M}}$, composed of a number $M$ of identical subsystems $\left(X_{1}\right)$ with spin number $S_{X_{1}}$, it holds

$$
\epsilon\left(X_{M}\right)=M\left[\epsilon\left(X_{1}\right)-2\left(\frac{S_{X_{M}}}{M}-S_{X_{1}}\right)\right] \geq M \epsilon\left(X_{1}\right) .
$$

The equality is valid for the case in which the system dissociates into closed-shell subsystems ${ }^{40}$ and indicates that the cumulant of the system is equal to $M$ times the individual 2-RDM cumulants, and for open-shell states that couple in such a way that the term $\left(S_{X_{M}} / M\right)-S_{X_{1}}$ vanishes (an example will be shown in the next section), while the inequality holds for other coupling cases of open-shell fragments. A similar expression may be derived in the case the subsystems are not identical. Therefore, the inequality measures the correlation of the subsystems due to the spin entanglement and the size consistency (extensivity) property applies to CCTM because the cumulant scales linearly with the size of the system.

In the next section we report numerical determinations which guarantee the reliability of our proposal.

\section{RESULTS AND DISCUSSION}

Numerical calculations have been performed for selected systems in order to achieve a reliable physical understanding of the concept of electron correlation and entanglement. Two situations have been considered: molecular systems in equilibrium conformations and dissociation processes that represent conformations out of equilibrium. For the systems in equilibrium, the calculations have been carried out at their corresponding experimental geometries, ${ }^{51}$ at level of configuration interaction with single and double excitations (CISD), using 6-31G basis sets. For the dissociation sequences a full configuration interaction level has been used with minimal basis (STO-3G) sets to render the calculations feasible. All calculations have been performed with the PSI 3.3 program package $^{52}$ using as reference the restricted Hartree-Fock wave functions (singlets) and restricted openshell Hartree-Fock (doublets and triplets) states and their corresponding molecular orbitals as orthonormal basis sets. 
TABLE I. CCTM $\epsilon$, number of electrons $N$, and spin number $S$ for selected species at their equilibrium conformation, at configuration interaction level (single and double excitations), with 6-31G basis sets.

\begin{tabular}{lcccc}
\hline \hline System & State & $\epsilon$ & $N$ & $S$ \\
\hline $\mathrm{HF}$ & ${ }^{1} \Sigma^{+}$ & 0.2372 & 10 & 0 \\
$\mathrm{H}_{2} \mathrm{O}$ & ${ }^{1} A_{1}$ & 0.3081 & 10 & 0 \\
$\mathrm{NH}_{3}$ & ${ }^{1} A_{1}$ & 0.3520 & 10 & 0 \\
$\mathrm{CH}_{4}$ & ${ }^{1} A_{1}$ & 0.3858 & 10 & 0 \\
$\mathrm{C}_{2}$ & ${ }^{3} \Sigma_{g}^{+}$ & 0.5043 & 12 & 1 \\
$\mathrm{O}_{2}$ & ${ }^{3} \Sigma_{g}^{-}$ & 0.5036 & 16 & 1 \\
$\mathrm{HBBH}$ linear & ${ }^{3} \Sigma_{g}^{-}$ & 0.3981 & 12 & 1 \\
$\mathrm{CH}_{3}$ & ${ }^{2} A_{1}$ & 0.3118 & 9 & $\frac{1}{2}$ \\
$\mathrm{CH}_{2}$ & ${ }^{3} B_{1}$ & 0.2414 & 8 & 1 \\
$\mathrm{CH}^{2}$ & ${ }^{2} \Pi$ & 0.3499 & 7 & $\frac{1}{2}$ \\
$\mathrm{NO}$ & ${ }^{2} \Pi$ & 0.5480 & 15 & $\frac{1}{2}$ \\
$\mathrm{H}_{2} \mathrm{NO}$ & ${ }^{2} B_{1}$ & 0.5080 & 17 & $\frac{1}{2}$ \\
\hline \hline
\end{tabular}

Table I reports the results for the CCTM $\epsilon$ and related quantities for several molecular systems at their equilibrium conformations. We will discuss the systems in that Table divided into selected compound sets to relate the concept of correlation with global properties of physical relevance. The first set is composed by the isoelectronic series constituted by the molecules $\mathrm{HF}, \mathrm{H}_{2} \mathrm{O}, \mathrm{NH}_{3}$, and $\mathrm{CH}_{4}$ in their singlet states; as may be seen in Table I, the CCTM $\epsilon$ increases as the system becomes linked by stronger covalent bonds, i.e., following the sequence $\mathrm{HF}<\mathrm{H}_{2} \mathrm{O}<\mathrm{NH}_{3}<\mathrm{CH}_{4}$. These results are physically acceptable because ionic systems tend to transfer charge from one atom to another to preserve a closed-shell structure, while covalent bonds increase the charge density in the internuclear regions. ${ }^{6,7}$ The second set is composed by the diatomic homonuclear molecules $\mathrm{C}_{2}, \mathrm{O}_{2}$ and the linear $\mathrm{HBBH}$ one in their triplet ground states, possessing all of them an inversion center; as can be observed all these systems show similar CCTM despite the number of electrons of the diatomic systems is different. These results are consistent with those observed in the first series because in these homonuclear molecules each atom possesses an even number of electrons providing a covalent bond and $\mathrm{BH}$ and $\mathrm{BB}$ bonds in $\mathrm{HBBH}$ are covalent. ${ }^{35}$ The group of systems composed of carbon and hydrogen atoms $\mathrm{CH}_{4}, \mathrm{CH}_{3}, \mathrm{CH}_{2}, \mathrm{CH}$ is useful to compare the effect the number of substates of a multiplet has on the correlation of the system. The $\mathrm{CH}_{4}$ singlet possesses the greater $\epsilon$ within this set. The CCTM decreases as the multiplicity of the state increases having similar values for the $\mathrm{CH}_{3}$ and $\mathrm{CH}$ doublets and smaller value for the triplet state of $\mathrm{CH}_{2}$. Another group to exemplify the equilibrium situations is that of doublet states of $\mathrm{NO}$ and $\mathrm{H}_{2} \mathrm{NO}$ which have similar CCTM values, showing the same trend as the above hydrocarbon series. All these simple examples have permitted to note that the spin projection $S$ and the number of electrons in the system are relevant to describe such a correlation measure but also the nature of the bonds is important to give a physical insight about this property.

The second step to test the CCTM proposal is to consider its behavior in molecular conformations out of equilibrium, i.e., electron rearrangements during chemical reactions.
TABLE II. Size consistency of the CCTM $\epsilon$, for molecular systems at infinite internuclear distances, at full configuration interaction level, with STO-3G basis sets.

\begin{tabular}{lcc}
\hline \hline System & State & $\epsilon$ \\
\hline $\mathrm{Be}_{2}$ & ${ }^{1} \Sigma_{g}^{+}$ & 1.553 \\
$2 \mathrm{Be}$ & & 1.553 \\
$\mathrm{H}_{2}$ & ${ }^{1} \Sigma_{g}^{+}$ & 2.000 \\
$2 \mathrm{H}$ & & 0.000 \\
$\mathrm{Li}_{2}$ & ${ }^{1} \Sigma_{g}^{+}$ & 2.002 \\
$2 \mathrm{Li}$ & & 0.002 \\
$\mathrm{Li}_{2}$ & ${ }^{3} \Sigma_{g}^{+}$ & 0.002 \\
$2 \mathrm{Li}$ & & 0.002 \\
$\mathrm{H}_{4}$ (chain) & ${ }^{1} \Sigma_{g}^{+}$ & 4.000 \\
$4 \mathrm{H}$ & & 0.000 \\
$\mathrm{H}_{6}$ (chain) & ${ }^{1} \Sigma_{g}^{+}$ & 6.000 \\
$6 \mathrm{H}$ & & 0.000 \\
\hline \hline
\end{tabular}

To give a physical interpretation of the present measure we have chosen dissociation processes of diatomic molecular systems in states which separate into both closed- or openshell fragments. The size consistency of this measure is of fundamental importance to test its ability to quantify spin entanglement, as noted in Ref. 22. Table II and Figs. 1-6 show the behavior of $\epsilon$ in dissociation processes. Let us inspect the results regarding the consistency reference of CCTM $\epsilon$ for a system such that $M$ times the measure of an isolated single closed-shell fragment (atom or group of atoms) equals $M$ times the measure of one of these closedshell fragments at infinite separation. Table II shows that the CCTM $\epsilon$ for dissociation of the $\mathrm{Be}_{2}$ molecule at infinite nuclear separation equals the measure for two isolated Be atoms because both the molecule and the atoms possess closed-shell structure. Figure 1 shows that the $\epsilon$ value for the system $\mathrm{Be}_{2}$ asymptotically tends to the corresponding value for two isolated Be atoms. This observation points out that the correlation only arises from the interaction of the electrons inside each fragment. Figure 1 shows the increment of $\mathrm{Be}_{2}$ correlation energy $E_{\text {corr }}$ as a function of the interatomic distance, reaching a constant value for infinite separation. It may be noted that absolute values of $E_{\text {corr }}$ and CCTM $\epsilon$ show

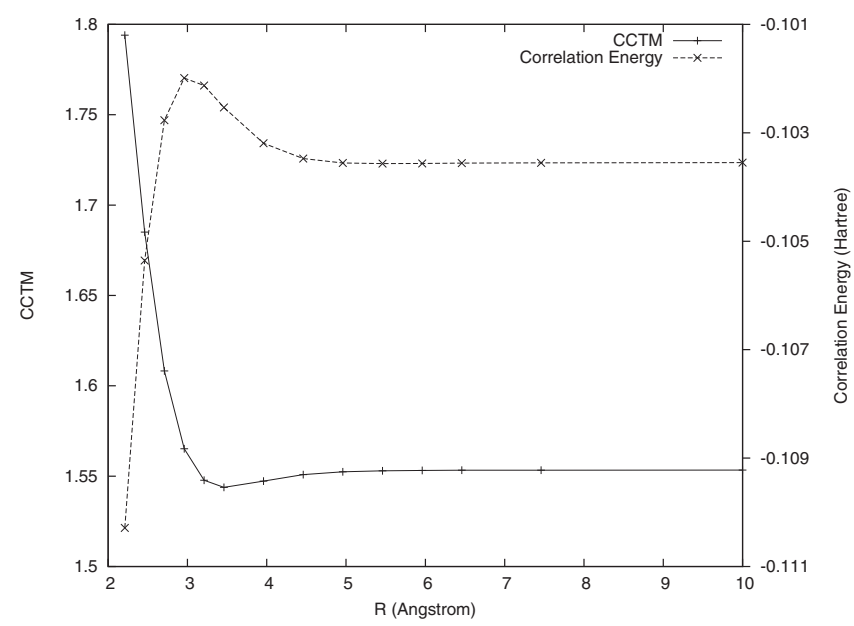

FIG. 1. CCTM $\epsilon$ and correlation energy, $E_{\text {corr }}$, as functions of the internuclear distance $R$ (in angstroms) for $\mathrm{Be}_{2}$ molecule in singlet ground state. 


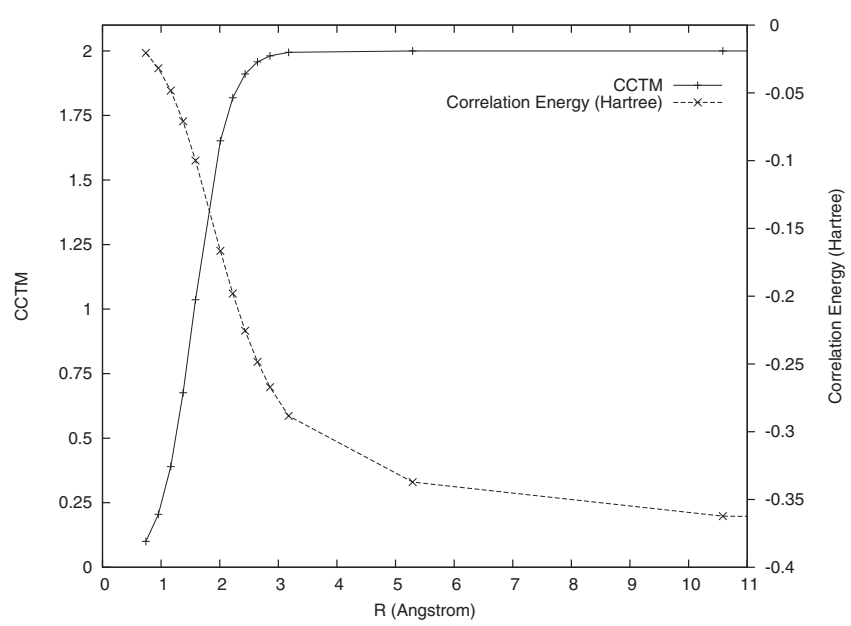

FIG. 2. CCTM $\epsilon$ and correlation energy, $E_{\text {corr }}$, as functions of the internuclear distance $R$ (in angstroms) for $\mathrm{H}_{2}$ molecule in singlet ground state.

a similar behavior during the dissociation process. The $\mathrm{H}_{2}$ system in singlet state is crucial to understand dissociation processes into open-shell fragments that couple into a singlet state. For infinite distances, $\epsilon\left(\mathrm{H}_{2}\right)=2.0$, while for isolated hydrogen atoms $\epsilon(\mathrm{H})=0$ because there is no electron interactions, as shown in Table II. Therefore, the only source of correlation in this system is due to spin, i.e., spin entanglement. Figure 2 shows the increase in correlation energy and CCTM $\epsilon$ due to the spin state of the system. To complement this example, the $\mathrm{Li}_{2}$ molecule in its singlet state is presented, to show the CCTM when a larger number of electrons is considered. Figure 3 shows $\epsilon\left(\mathrm{Li}_{2}\right)$ reaching the value 2.002 at infinite nuclear separation and Table II shows that $\epsilon\left(\mathrm{Li}_{2}\right)>2 \epsilon(\mathrm{Li})>0$. This latter result reflects the fact that as Li fragments are open-shell atoms, the correlation is not only due to spin but also to correlation effects of the electrons in the fragments. Thus, the difference between the $\epsilon$ value of $\mathrm{Li}_{2}$ system at infinite internuclear separation and that corresponding to two infinitely separated Li atoms measures entanglement due to spin. Figure 3 shows that the correlation energy increases following the same trend as the $\mathrm{H}_{2}$ system. However, $\mathrm{Li}_{2}$ in a triplet state, i.e., a greater spin multiplicity

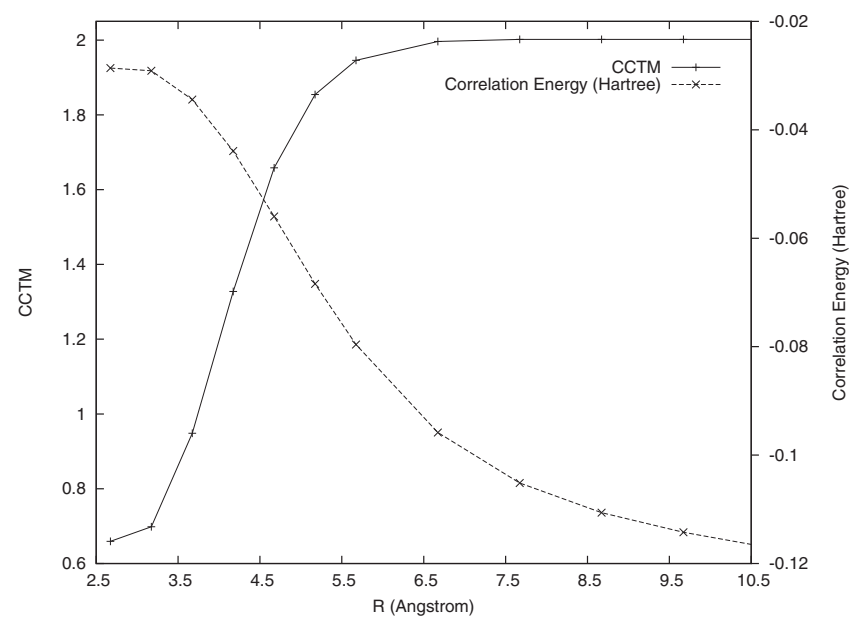

FIG. 3. CCTM $\epsilon$ and correlation energy, $E_{\text {corr }}$, as functions of the internuclear distance $R$ (in angstroms) for $\mathrm{Li}_{2}$ molecule in singlet ground state.

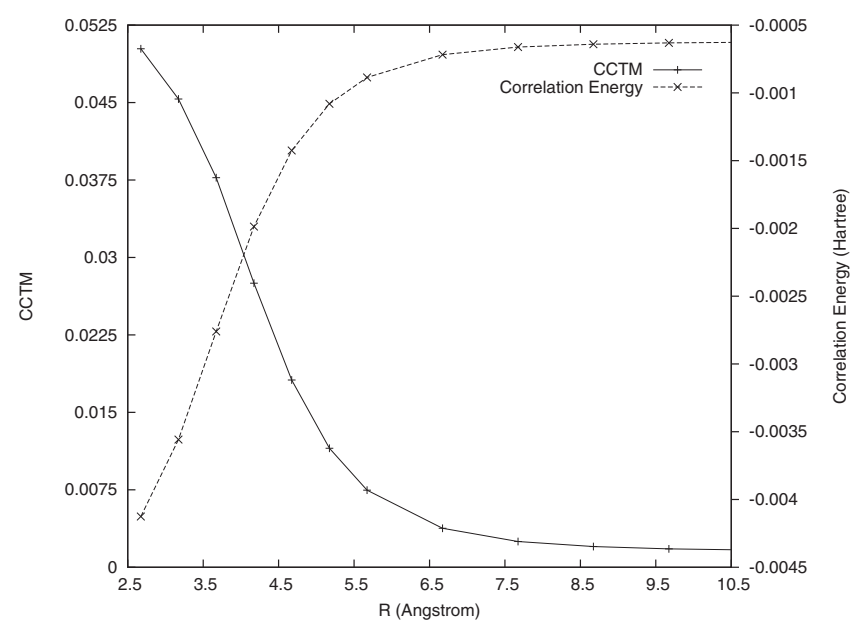

FIG. 4. CCTM $\epsilon$ and correlation energy, $E_{\text {corr }}$, as functions of the internuclear distance $R$ (in angstroms) for $\mathrm{Li}_{2}$ molecule in triplet state.

of the state (cf. Table II), shows that at infinite distance $\epsilon\left(\mathrm{Li}_{2}\right)=2 \epsilon(\mathrm{Li})$, because the unpaired electrons accommodate in different spatial parts; i.e., the wave function of the triplet is expanded nearly by only one Slater determinant at all distances leading to a nonentangled spin state for large distances between the nuclei. This situation is different from the singlet state which needs at least two Slater determinants to be expanded (Fig. 4). It means that this correlation measure is also capable of detecting the deviation of the wave function of the system from a monodeterminantal one and provides a measure of the multiconfigurational character of the wave function. Figure 4 shows the vanishing correlation energy for the infinitely far apart fragments. The last set of systems is two $\mathrm{H}$ atom chains, $\mathrm{H}_{4}$ and $\mathrm{H}_{6}$ in their singlet states, i.e., $\mathrm{H}-\mathrm{H}-\mathrm{H} \cdots \mathrm{H}$ which show results for CCTM and correlation energy similar to those obtained for $\mathrm{H}_{2}$ singlet state (cf. Fig. 5). Table II shows CCTM values 4.0 and 6.0 respectively; due to the absence of electron interaction within the fragments, these CCTM values arise only from spin entanglement in both systems. Similarly, the correlation energies increase as the internuclear distance between the atoms tends to be infinite, as shown in Figs. 5 and 6.

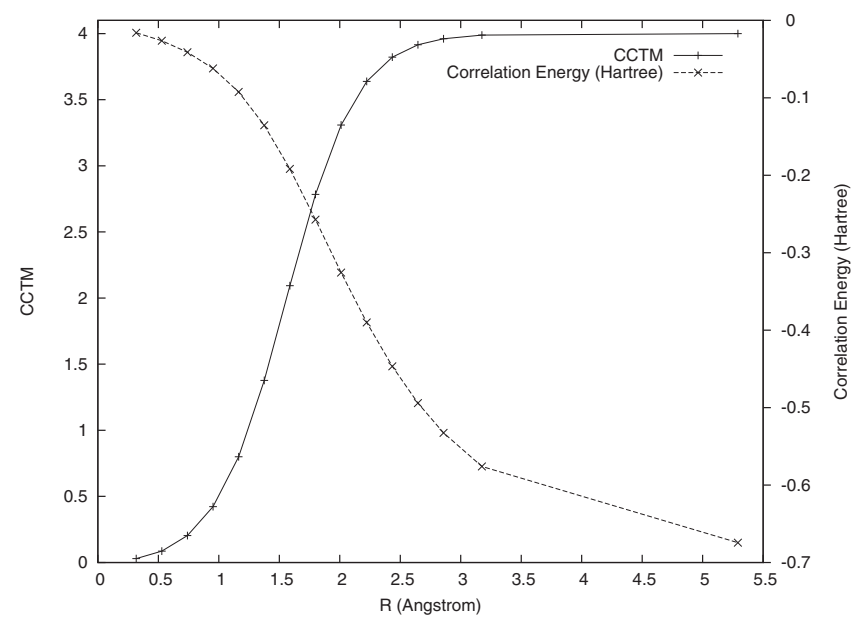

FIG. 5. CCTM $\epsilon$ and correlation energy, $E_{\text {corr }}$, as functions of the internuclear distance $R$ (in angstroms) for $\mathrm{H}_{4}$ molecule in ground singlet states. 


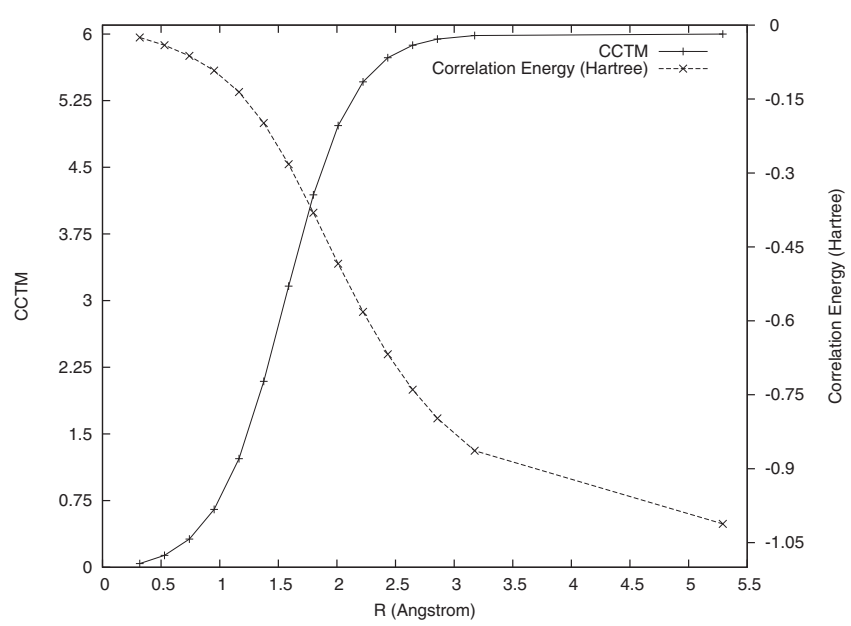

FIG. 6. CCTM $\epsilon$ and correlation energy, $E_{\text {corr }}$, as functions of the internuclear distance $R$ (in angstroms) for $\mathrm{H}_{6}$ molecule in ground singlet states.

\section{CONCLUDING REMARKS}

In this work we have dealt with electron correlation effects, which are of paramount importance to describe molecular properties and electron rearrangement. We have focused on the entanglement concept, that is, the correlation in a quantum system which has no classical picture. We have proposed a measure of electron correlation and entanglement within the spin-free formulation of quantum chemistry. We have also analyzed the physical and mathematical properties of that quantity, which has been termed by the acronym CCTM. This measure which is directly related with the 2-RDM many-body cumulant allows one to interpret the onset of the contributions to the correlation effects and to evaluate the spin entanglement, which is an important part of such correlation effects. Numerical examples are presented dealing with equilibrium and nonequilibrium geometrical structures in selected molecular systems to measure spin correlations. The present proposal, i.e., CCTM, can be applied to any wave function quality since it does not depend on how the 2-RDM is obtained. The information contained in the 2-RDM and the size consistency of its many-body cumulant indicate that the CCTM quantity is a useful tool to properly describe the strength of electron correlation and entanglement in quantum mechanics.

\section{ACKNOWLEDGMENTS}

This report has been financially supported by Projects X017 (Universidad de Buenos Aires), PIP No. 11220090100061 (Consejo Nacional de Investigaciones Científicas y Técnicas, República Argentina), Grant No. CTQ2009-07459/BQU (the Spanish Ministry of Education) and Grant No. GIU09/43 (Universidad del País Vasco). We thank the Universidad del País Vasco for allocation of computational resources.

${ }^{1}$ P. Fulde, Electron Correlations in Molecules and Solids (Springer, Berlin, 1993) (and references therein).

${ }^{2}$ P. O. Löwdin, Phys. Rev. 97, 1474 (1955).

${ }^{3}$ E. R. Davidson, Reduced Density Matrices in Quantum Chemistry (Academic, New York, 1976) (and references therein)

${ }^{4}$ R. F. W. Bader, Atoms in Molecules: A Quantum Theory (Clarendon,
Oxford, 1994) (see also references therein).

${ }^{5}$ P. L. A. Popelier, Atoms in Molecules: An Introduction (Pearson, London, 1999).

${ }^{6}$ R. M. Lobayan, R. C. Bochicchio, L. Lain, and A. Torre, J. Chem. Phys. 123, 144116 (2005).

${ }^{7}$ R. M. Lobayan, R. C. Bochicchio, L. Lain, and A. Torre, J. Phys. Chem. A 111, 3166 (2007).

${ }^{8}$ R. M. Lobayan, R. C. Bochicchio, A. Torre, and L. Lain, J. Chem. Theory Comput. 5, 2030 (2009)

${ }^{9}$ R. M. Lobayan, D. R. Alcoba, R. C. Bochicchio, A. Torre, and L. Lain, J. Phys. Chem. A 114, 1200 (2010).

${ }^{10} \mathrm{R}$. McWeeny, Methods of Molecular Quantum Mechanics (Academic, London, 1969) (see also references therein).

${ }^{11}$ A. Szabo and N. S. Ostlund, Modern Quantum Chemistry: Introduction to Advanced Electronic Structure (Macmillan, New York, 1982).

${ }^{12}$ R. C. Bochicchio, J. Mol. Struct.: THEOCHEM 228, 209 (1991) (and references therein).

${ }^{13}$ X. Fradera, M. A. Austen, and R. F. W. Bader, J. Phys. Chem. A 103, 304 (1999).

${ }^{14}$ R. C. Bochicchio, L. Lain, and A. Torre, Chem. Phys. Lett. 374, 567 (2003).

${ }^{15}$ D. R. Alcoba, R. C. Bochicchio, L. Lain, and A. Torre, Chem. Phys. Lett. 442, 157 (2007).

${ }^{16}$ K. Takatsuka, T. Fueno, and K. Yamaguchi, Theor. Chim. Acta 48, 175 (1978).

${ }^{17}$ K. Takatsuka and T. Fueno, J. Chem. Phys. 69, 661 (1978).

${ }^{18}$ R. C. Bochicchio, J. Mol. Struct.: THEOCHEM 429, 229 (1998).

${ }^{19}$ R. C. Bochicchio, L. Lain, and A. Torre, Chem. Phys. Lett. 375, 45 (2003).

${ }^{20}$ A. V. Luzanov and O. V. Prezhdo, Mol. Phys. 105, 2879 (2007).

${ }^{21}$ A. V. Luzanov and O. A. Zhikol, Int. J. Quantum Chem. 104, 167 (2005).

${ }^{22}$ T. Juhász and D. A. Mazziotti, J. Chem. Phys. 125, 174105 (2006) (and references therein).

${ }^{23} \mathrm{~S}$. Kais, in Reduced-Density-Matrix Mechanics with Applications to Many-Electron Atoms and Molecules, Advances in Chemical Physics Vol. 134, edited by D. A. Mazziotti (Wiley-Intersience, Hoboken, 2007) (and references therein).

${ }^{24}$ W. Kutzelnigg and D. Mukherjee, J. Chem. Phys. 116, 4787 (2002).

${ }^{25}$ W. Kutzelnigg and D. Mukherjee, J. Chem. Phys. 110, 2800 (1999).

${ }^{26}$ L. Diósi, A Short Course in Quantum Information Theory. An Approach from Theoretical Physics (Springer, Berlin, 2007).

${ }^{27}$ G. C. Ghirardi, L. Marinatto, and T. Weber, J. Stat. Phys. 108, 49 (2002).

${ }^{28}$ G. C. Ghirardi and L. Marinatto, Opt. Spectrosc. 99, 386 (2005).

${ }^{29}$ R. McWeeny, Faraday Discuss. 135, 13 (2007).

${ }^{30}$ L. Lain, A. Torre, D. R. Alcoba, and R. C. Bochicchio, Chem. Phys. Lett. 476, 101 (2009).

${ }^{31}$ D. R. Alcoba, R. C. Bochicchio, L. Lain, and A. Torre, Chem. Phys. Lett. 429, 286 (2006).

${ }^{32} \mathrm{C}$. Valdemoro, in Reduced-Density-Matrix Mechanics with Applications to Many-Electron Atoms and Molecules, Advances in Chemical Physics Vol. 134, edited by D. A. Mazziotti (Wiley-Intersience, Hoboken, 2007) (and references therein).

${ }^{33}$ D. A. Mazziotti, Phys. Rev. A 57, 4219 (1998).

${ }^{34}$ D. A. Mazziotti, Chem. Phys. Lett. 289, 419 (1998).

${ }^{35}$ D. R. Alcoba, R. C. Bochicchio, L. Lain, and A. Torre, Phys. Chem. Chem. Phys. 10, 5144 (2008).

${ }^{36}$ R. McWeeny and Y. Mizuno, Proc. R. Soc. London A259, 554 (1961).

${ }^{37}$ L. Lain, A. Torre, and R. Bochicchio, J. Chem. Phys. 117, 5497 (2002).

${ }^{38}$ A. Torre and L. Lain, J. Mol. Struct.: THEOCHEM 426, 25 (1998) (and references therein).

${ }^{39}$ W. Kutzelnigg, in Reduced-Density-Matrix Mechanics with Applications to Many-Electron Atoms and Molecules, Advances in Chemical Physics Vol. 134, edited by D. A. Mazziotti (Wiley-Intersience, Hoboken, 2007) (and references therein).

${ }^{40} \mathrm{~J}$. Harriman, in Reduced-Density-Matrix Mechanics with Applications to Many-Electron Atoms and Molecules, Advances in Chemical Physics Vol. 134, edited by D. A. Mazziotti (Wiley-Intersience, Hoboken, 2007).

${ }^{41}$ D. R. Alcoba, L. Lain, A. Torre, and R. C. Bochicchio, Chem. Phys. Lett. 470, 136 (2009).

${ }^{42}$ L. Lain, A. Torre, R. C. Bochicchio, and R. Ponec, Chem. Phys. Lett. 346, 283 (2001).

${ }^{43}$ V. N. Staroverov and E. R. Davidson, J. Am. Chem. Soc. 122, 186 (2000).

${ }^{44}$ V. N. Staroverov and E. R. Davidson, Chem. Phys. Lett. 330, 161 (2000). 
${ }^{45}$ A. Torre, D. R. Alcoba, L. Lain, and R. C. Bochicchio, J. Phys. Chem. A 114, 2344 (2010).

${ }^{46}$ M. Head-Gordon, Chem. Phys. Lett. 372, 508 (2003).

${ }^{47}$ R. C. Bochicchio, A. Torre, and L. Lain, Chem. Phys. Lett. 380, 486 (2003).

${ }^{48}$ M. Rosina, in Reduced Density Matrices with Applications to Physical and Chemical Systems, Queen's Papers on Pure and Applied Mathematics Vol. 11, edited by J. Coleman and R. M. Erdahl (Queen's University,
Kingston, Ontario, 1968).

${ }^{49}$ D. Bru $\beta$, J. Math. Phys. 43, 4237 (2002).

${ }^{50}$ C. Valdemoro, Phys. Rev. A 45, 4462 (1992).

${ }^{51}$ Handbook of Chemistry and Physics, 59th ed., edited by R. C. Weast (CRC, Cleveland, OH, 1979).

${ }^{52}$ T. D. Crawford, C. D. Sherrill, E. F. Valeev, J. T. Fermann, R. A. King, M. L. Leininger, S. T. Brown, C. L. Janssen, E. T. Seidl, J. P. Kenny, and W. D. Allen, J. Comput. Chem. 28, 1610 (2007). 